TRABAJO DE INVESTIGACIÓN

\title{
No erupción de segundos molares mandibulares permanentes en radiografía panorámica.
}

\section{Unerupted mandibular permanent second molars in Radiographic analysis}

\begin{abstract}
Betty Bizcar ${ }^{1}$, Pablo Navarro², Paulo Sandoval ${ }^{3}$
1. Programa de Magister en Odontología, Facultad de Odontología, Universidad de La Frontera, Temuco, Chile.

2. Bioestadístico del Centro de Investigación en Ciencias Odontológicas, Facultad de Odontología, Universidad de La Frontera, Temuco, Chile.

3. Dirección de Postgrado, Facultad de

Odontología, Universidad de La Frontera, Temuco, Chile.

Correspondencia a: Prof. Paulo Sandoval Vidal. | Departamento de Odontopediatría y Ortodoncia, Facultad de Odontología Universidad de la Frontera | Manuel Montt 115, Temuco, CHILE | Email: paulo. sandoval@ufrontera.cl

Trabajo recibido el 21/08/2016. Aprobado para su publicación el 27/11/2016

\section{RESUMEN}

La no erupción de segundos molares es relativamente baja, pero ha ido en aumento en los últimos años. Se relaciona con un tratamiento multidisciplinario largo, costoso y complejo, por lo cual es de suma importancia enfocarse en el diagnóstico precoz y tratamiento temprano. El objetivo de este estudio es poder hacer una comparación de medidas radiográficas entre un grupo de pacientes con segundo molar mandibular permanente no erupcionado o sin posibilidades de erupcionar y un grupo control. Método: Se realizó una revisión manual consecutiva de las 16.835 fichas sobre las cuales se aplicaron criterios de inclusión y exclusión. Los datos se obtuvieron de radiografías panorámicas de las cuales se obtuvieron medidas lineales y angulares que fueron comparados entre dos grupos de estudio. Se desarrolló un modelo predictivo mediante análisis discriminante.

Resultados: El 1,5\% de los pacientes tuvieron segundos molares no erupcionados. Los promedios de las variables relevantes fueron: Ángulo del segundo molar $33,7^{\circ}$; distancia desde distal del primer molar al borde anterior de la rama mandibular $15 \mathrm{~mm}$; invasión distal del primer molar por el segundo molar 2,1mm; inclinación mesial de segundo molar con respecto al plano oclusal $128^{\circ}$ y al plano mandibular $63^{\circ}$. El puntaje crítico discriminante fue de 36,7 .

Conclusión: La presencia de segundos molares incluidos es baja. Se encontró diferencias estadísticamente significativas entre casos y controles en todas las variables. El modelo predictivo puede identificar molares sin probabilidad de erupción natural. Es necesario contar con nuevos estudios con muestras poblacionales para establecer cuáles serían los valores de normalidad para poder diagnosticar en forma precoz esta condición.
\end{abstract}

\section{PALABRAS CLAVE}

Diente impactado; Diente incluido; molares permanentes.

Rev. Clin. Periodoncia Implantol. Rehabil. Oral Vol. 10(1); 44-48, 2017.

\section{ABSTRACT}

No eruption of seconds is relatively low, has been increasing in recent years. It relates to long, costly and complex multidisciplinary treatment, so it is important to focus on early diagnosis and early treatment. The aim of this study is to make a comparison of radiographic measurements among a group of patients with mandibular second permanent molar eruption and no possibility of a control group.

Method: A consecutive manual review of 16,835 sheets on which inclusion and exclusion criteria were applied. The data were obtained from panoramic radiographs of which linear and angular measurements were compared between two study groups were obtained. A predictive model was developed using discriminant analysis.

Results: $1.5 \%$ of patients had unerupted second molars. The averages of the relevant variables were: Angle of the second molar $33.7^{\circ}$; distance from distal of the first molar to the anterior border of the ramus $15 \mathrm{~mm}$; invasion distal of the first molar the second molar $2.1 \mathrm{~mm}$; second molar mesial inclination relative to the occlusal plane $128^{\circ}$ and $63^{\circ}$ mandibular plane. The critical discriminant score was 36.7 .

Conclusion: Statistically significant differences between cases and controls was found in all variables. The predictive model can identify likely molars with or without natural eruption. It is necessary to have further studies with population samples to establish what would be the normal values to diagnose this condition at an early stage.

KEY WORDS

Tooth, Impacted; Tooth, Unerupted; permanent molars.

Rev. Clin. Periodoncia Implantol. Rehabil. Oral Vol. 10(1); 44-48, 2017. 
No erupción de segundos molares mandibulares permanentes en radiografía panorámica.

\section{INTRODUCCIÓN}

La no erupción del segundo molar mandibular permanente es una condición relativamente baja, pero ha ido en aumento en los últimos años $^{1,2,3,4,5}$. La Dra. Evans fue la primera en publicar sobre este tema y la medida más importante que ella relacionó con esta condición fue la medida del ángulo del segundo molar mandibular permanente, formado entre los ejes axiales del $1^{\circ}$ molar y el $2^{\circ}$ molar (2MM), los valores fueron entre $65^{\circ}$ y $15^{\circ}$ con un promedio de $25^{\circ}$ grados ${ }^{1}$ Posteriormente otros autores han incluido otras medidas en e análisis de radiografías panorámicas con el propósito de diagnosticar e intervenir de forma temprana. Variables como la distancia desde distal del primer molar al borde anterior de la rama mandibular, tamaño del ángulo formado entre el eje axial del segundo molar con el plano mandibular ${ }^{2}$. También se ha relacionado la no erupción del segundo molar con sexo, edad, presencia de terceros molares, pérdidas prematuras de dientes temporales, uso de aparatos para ganar espacio en el sector anterior ${ }^{1,3,4,5}$. La retención de segundos se relaciona también con tratamientos más largo, costosos y complejos ${ }^{6}$ $7,8,9$, por lo cual es de suma importancia enfocarse en el diagnóstico precoz ${ }^{10}$ y tratamiento temprano. El objetivo de este estudio es pode hacer una comparación de medidas radiográficas entre un grupo de pacientes con segundo molar mandibular permanente no erupcionado o sin posibilidades de erupcionar, por presentar un ángulo de $2 \mathrm{MM}$ mayor a $25^{\circ}$ y un grupo control.

\section{MATERIAL Y MÉTODO}

Se tuvo acceso a todas las fichas entre los años 2004 a 2012 de población general que consulta por atención en la Clínica Odontológica Docente Asistencial (CODA) de la Universidad de La Frontera, de la ciudad de Temuco, Chile. Se realizó una revisión manual consecutiva de las 16.835 fichas. Los criterios de inclusión para este estudio fueron edad entre 8 y 16 años, disponibilidad de una radiografía panorámica análoga tomada entre las edades antes mencionadas. Criterios de exclusión fueron presentar patología sindrómica, agenesias, supernumerarios, segundos molares con inclinación distal. Luego de aplicados estos criterios se obtuvieron 1.353 fichas a las cuales se les midió el ángulo del segundo molar. Si era mayor o igual a $25^{\circ}$ se consideraron sin posibilidades de erupción exitosa y fueron incluidos en el grupo de los casos $(\mathrm{N}=25)$. De los 1.328 que presentaron ángulo menor a $25^{\circ}$ se obtuvo el grupo control. Por cada caso se seleccionaron 3 controles ${ }^{11}$ de forma aleatorizada, durante las mediciones se perdieron los datos de 3 individuos del grupo contro $(\mathrm{N}=72)$ por tener solo una hemiarcada posible de medir, por lo que fueron eliminados.

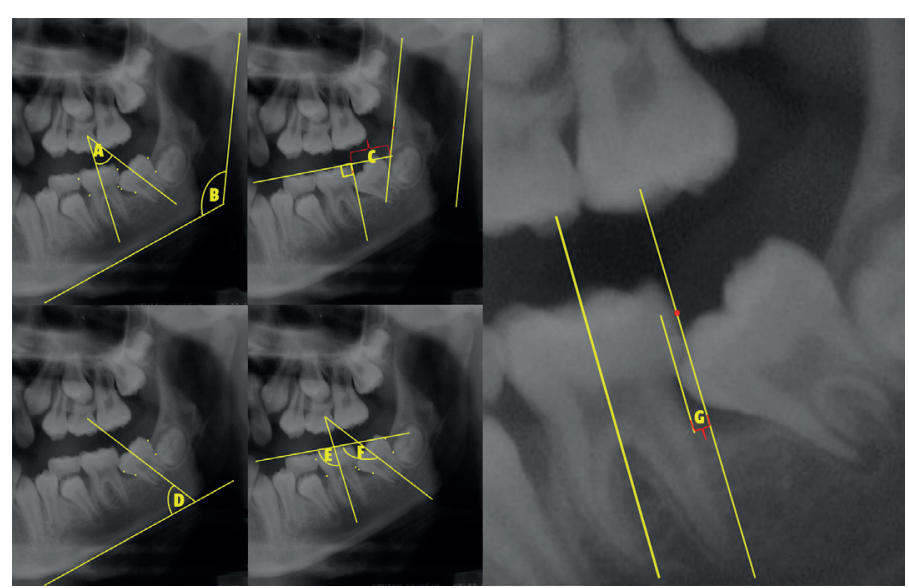

Figura 1. Mediciones en radiografía panorámica: (a) Angulo del segundo molar (b) Angulo goniaco (c) Distancia del primer molar al borde anterior de la rama (d) Angulo del primer molar con plano mandibular (e) Angulo del primer molar con plano oclusal (f) Angulo del segundo molar con plano oclusal (g) Invasión de segundo molar por distal del primer molar mandibular.

Las variables estudiadas en este trabajo fueron la medida del ángulo $2 \mathrm{MM}$, como lo midió Evans ${ }^{1}$ y se le incorporaron puntos coronales para objetivar la medición descritos por Rubin et al. ${ }^{12}$ (Fig. 1a). Se midió el ángulo goniaco (panorámico) trazando una línea al borde inferior del cuerpo mandibular y una tangente al borde posterior de la rama similar al trazado cefalométrico (Fig. 1b). Otra variable fue la distancia del primer molar (MM1) al borde anterior de la rama, para lo cual se trazó la línea del plano oclusal (PO) hasta la rama, se marcó una línea perpendicular al plano oclusal que pase por el punto más distal de la corona de MM1, a continuación se trazó una línea al borde posterior de la rama similar a la usada para medir el ángulo mandibular, después se marcó un punto en la parte más cóncava del borde anterior de la rama y se trazó una línea hasta el PO que pasara por este punto y que fuera paralela a la línea posterior de la rama. Finalmente la distancia es medida en milímetros sobre la línea del PO entre la línea vertical por distal del primer molar antes mencionadas y la línea vertical al borde anterior de la rama (Fig. 1c). Para medir el ángulo entre MM2 y plano mandibular se usó la línea del eje longitudinal descrito por Rubin et al. ${ }^{12}$ de MM2 y se extendió hasta la línea del plano mandibular, se midió el ángulo por anterior (fig. 1d). Para medir el ángulo de MM1 con PO, se usó el eje longitudinal del MM1 descrito por Rubin et al. ${ }^{12}$ y se extendió hasta el $\mathrm{PO}$, el ángulo se midió por anterior (Fig. 1e). El ángulo MM2 y $\mathrm{PO}$ se obtuvo de la misma forma que el anterior usando el eje longitudinal del MM2 (Fig. 1f). La invasión de MM2 por distal del MM1, fue medida trazando una línea paralela al eje longitudinal de MM1 que pasara por el punto coronal más distal de $\mathrm{MM1}$ y se midió en milímetros la cantidad de MM2 que se encontrara por delante de esta línea (Fig. 1g). Se observó la presencia o ausencia de terceros molares independiente de la etapa de desarrollo en la que se encontrara.

Se usó un negatoscopio, radiografías panorámicas análogas, papel de acetato (cefalométrico) sobre el cual se trazaron líneas y ángulos con lápiz de grafito con una mina de $0,5 \mathrm{~mm}$ los que fueron medidos con un goniómetro (Baseline TM CE, Diagnostic and Measuring Instruments, USA) por un operador el cual realizó un proceso de calibración con un ortodoncista experto. Para corroborar la fiabilidad de los datos se usó un coeficiente de correlación intraclase $(\mathrm{CCI})$ Fleiss y Cohen ${ }^{13}$ la que dio un valor de concordancia de 0,997 $(>0,90$ muy buena) las mediciones se realizaron dos veces con intervalo de 7 días entre cada medición.

\section{Análisis estadístico.}

La recolección de los datos fue registrada en una planilla Office Excel (Microsoft, CA, USA). Se realizó la prueba de normalidad de Kolgomorov-Smirnov, Prueba t para muestras Independientes y prueba de U-Mann-Whitney. Un valor de $p<0,05$ se eligió como umbral de la significación estadística.

El análisis discriminante fue aplicado para los 52 molares sin posibilidad de erupción. Para lograr el mejor modelo de discriminación, en la primera fase del análisis fueron seleccionadas las variables más importantes para pertenecer al grupo casos o al grupo control. Luego un procedimiento paso a paso fue utilizado para identificar una "buena" variable predictora. Siguiendo la selección con el valor $\mathrm{F}$, se eligió el valor 4 para entrar o para salir del grupo. Cuando se determinó el menor grupo de variables discriminantes significativas, el poder predictivo (poder clasificativo) del modelo fue probado mediante el análisis discriminante. Este último procedimiento provee un método para pronosticar dentro de un grupo a un nuevo paciente y que tenga mayor posibilidad que entre correctamente. Luego se calculó el Coeficiente de función discriminante no estandarizada para cada variable previamente seleccionada, este cálculo conduce a una ecuación que asigna un puntaje a cada paciente. Una media de puntaje para cada grupo se calcula y la mitad de ese puntaje es el valor crítico que establece a cuál de los dos grupos el paciente pertenece ${ }^{17,18}$.

Para el análisis de los datos se usó el programa estadístico SPSS Statistics for Windows (versión 20.0, IBM Corp., Armonk, NY).

\section{RESULTADOS}

La estadística descriptiva de las variables en estudio para casos y controles son mostrados en forma gráfica en las tablas 1. La distribución normal de los valores fue verificada en ambos grupos con el test de Kolgomorov-Smirnov. Destaca la invasión distal del segundo molar sobre la cara distal del primer molar permanente en que los casos tiene un rango entre uno a tres milímetros y en los controles no alcanza el medio milímetro. Esta es la primera variable del modelo que produce la más eficiente separación entre los dos grupos. Las otras variables explican más del $85 \%$ de la varianza, por lo que el coeficiente de función discriminante sin estandarizar de las variables (tabla 3 ) conduce a la siguiente ecuación que provee puntajes individuales para medir a cada paciente: 
Tabla 1. Análisis descriptivo de las variables medidas en 97 radiografías panorámicas.

\begin{tabular}{|c|c|c|c|c|}
\hline Variable & $\begin{array}{c}\text { Caso } \\
\text { Controles }\end{array}$ & $\mathbf{N}$ & Media & $\begin{array}{c}\text { Desviación } \\
\text { típica }\end{array}$ \\
\hline Angulo MM2 & $\begin{array}{l}\text { CASOS } \\
\text { CONTROLES }\end{array}$ & $\begin{array}{l}25 \\
72\end{array}$ & $\begin{array}{l}33,72^{\circ} \\
6,49^{\circ}\end{array}$ & $\begin{array}{l}6,25 \\
5,89\end{array}$ \\
\hline $\begin{array}{l}\text { Ángulo del MM2 } \\
\text { con plano mandibular }\end{array}$ & $\begin{array}{l}\text { CASOS } \\
\text { CONTROLES }\end{array}$ & $\begin{array}{l}25 \\
72\end{array}$ & $\begin{array}{l}62,96^{\circ} \\
90,57^{\circ}\end{array}$ & $\begin{array}{r}24,02 \\
6,77\end{array}$ \\
\hline $\begin{array}{l}\text { Angulo del MM2 } \\
\text { Con el plano oclusal }\end{array}$ & $\begin{array}{l}\text { CASOS } \\
\text { CONTROLES }\end{array}$ & 25 & $\begin{array}{l}127,72^{\circ} \\
100,56^{\circ}\end{array}$ & $\begin{array}{r}5,52 \\
11,87\end{array}$ \\
\hline $\begin{array}{l}\text { Invasión distal del MM1 } \\
\text { Por el MM2 }\end{array}$ & $\begin{array}{l}\text { CASOS } \\
\text { CONTROLES }\end{array}$ & $\begin{array}{l}25 \\
72\end{array}$ & $\begin{array}{l}2,08 \mathrm{~mm} \\
0,12 \mathrm{~mm}\end{array}$ & $\begin{array}{l}0,80 \\
0,21\end{array}$ \\
\hline $\begin{array}{l}\text { Distancia } 1^{\circ} \text { molar a } \\
\text { la rama mandibular }\end{array}$ & $\begin{array}{l}\text { CASOS } \\
\text { CONTROLES }\end{array}$ & $\begin{array}{l}25 \\
72\end{array}$ & $\begin{array}{l}14,96 \mathrm{~mm} \\
17,83 \mathrm{~mm}\end{array}$ & $\begin{array}{l}4,108 \\
4,104\end{array}$ \\
\hline $\begin{array}{l}\text { Angulo } 1^{\circ} \text { molar con } \\
\text { el plano oclusal }\end{array}$ & $\begin{array}{l}\text { CASOS } \\
\text { CONTROLES }\end{array}$ & 25 & $\begin{array}{l}94,84^{\circ} \\
95,56^{\circ}\end{array}$ & 4,317 \\
\hline Angulo goniaco & $\begin{array}{l}\text { CASOS } \\
\text { CONTROLES }\end{array}$ & 25 & $\begin{array}{l}128,44^{\circ} \\
127,93^{\circ}\end{array}$ & 4,975 \\
\hline
\end{tabular}

\begin{tabular}{|c|c|c|c|c|c|}
\hline & & & \multicolumn{2}{|c|}{ Frecuencia } & Porcentaje \\
\hline \multirow{4}{*}{ Presencia de tercer molar } & \multirow{2}{*}{ CASOS } & \multirow{2}{*}{25} & SI & 22 & $88 \%$ \\
\hline & & & NO & 3 & $12 \%$ \\
\hline & \multirow{2}{*}{ CONTROLES } & \multirow{2}{*}{72} & SI & 65 & $90,3 \%$ \\
\hline & & & NO & 7 & $9,7 \%$ \\
\hline
\end{tabular}

Puntaje (cada paciente) $=+2,0 \times$ (Invasión distal del MM1por el $\mathrm{MM} 2$ )

$+0,3 \times$ (Ángulo del segundo molar)

$+0,2 \times$ (Inclinación mesial de segundo molar con respecto al plano oclusal)

$+0,1 \times$ (Inclinación mesial de segundo molar con respecto al plano mandibular)

- 0,1 x (Distancia desde distal del primer molar al borde anterior de la rama mandibular)

Por la manera en que se interpreta la distancia del MM1 y la rama mandibular, a menor espacio existente menor es la probabilidad de erupción del MM2 en la ecuación se debe incluir con un valor negativo para que su registro aporte en el valor predictivo de manera correcta. Con todo esto el puntaje crítico, es decir el valor que divide entre casos y control fue de 36,7 .

\section{DISCUSIÓN}

El segundo molar mandibular es importante ya que participa en el soporte posterior, la eficiencia masticatoria, evita la elongación de su antagonista o como reemplazo del primer molar por pérdida prematura, a causa de caries o hipoplasia severa. Se ha considerado que es de suma importancia aportar datos científicos que nos permitan prevenir que el segundo molar mandibular no erupcione por una sobre inclinación mesial. Los resultados nos permiten decir que las variables medidas en este estudio se comportan de diferente forma entre casos y controles.

En relación al promedio del ángulo del segundo molar mandibular en este estudio se encontró un promedio de $33,7^{\circ}$, similar a lo observado por Cassetta et al. ${ }^{14}$ que fue $34,7^{\circ}$ en los casos, pero mayor a lo encontrado por Sonis y Ackerman15, el que fue de $24,6^{\circ}$, Rubin et al. ${ }^{7}$, quienes reportaron $17^{\circ}$ en los casos, pero en los controles obtuvieron un promedio de ángulo de $13,9^{\circ}$, mayor a lo observado en nuestro estudio, que fue de $6,4^{\circ}$. 
Tabla 2. Significancia de cada variable evaluada en radiografías panorámicas entre casos y controles.

Variables relacionadas

Casos - controles v/s ángulo MM2

$0,000^{*}$

Casos - controles v/s ángulo MM1 con plano

Oclusal

0,396

Casos - controles v/s $3^{\circ}$ molar

Casos - controles v/s ángulo goniaco

Casos - controles v/s distancia entre MM1 y la rama mandibular

$0,003^{*}$

Casos - controles v/s ángulo entre el eje axial

MM2 con el plano mandibular.

0,000 *

Casos - controles v/s ángulo entre el eje axial MM2 con el plano oclusal

$0,000^{*}$

Casos - controles v/s invasión distal MM1

0,000 *

*= Diferencias significativas

MM1 = Primer molar mandibula

$M M 2=$ Segundo molar mandibula

$\mathrm{V} / \mathrm{S}=$ versus

Tabla 3. Función discriminante

\begin{tabular}{lc}
\hline Variables predictivas & $\begin{array}{c}\text { Coeficiente de } \\
\text { función canónica } \\
\text { discriminante no } \\
\text { estandarizada }\end{array}$ \\
\hline Invasión distal del MM1 por el MM2 & 1,985 \\
Angulo MM2 & 0,286
\end{tabular}

Inclinación mesial de MM2con respecto al plano oclusal

Inclinación mesial de segundo molar con respecto al plano mandibular

Distancia desde distal del primer molar

al borde anterior de la rama mandibular

$0,098^{*}$

* Por el sentido en que se realiza la medición en la ecuación discriminante se le otorga un valor negativo.

Respecto a la medición de los milímetros que invade el segundo molar al primero, por distal, este estudio midió en promedio $2,08 \mathrm{~mm}$ en los casos, bastante mayor a lo encontrado por otros autores como Sonis y Ackerman ${ }^{15}$, que obtuvieron $0,57 \mathrm{~mm}$, y un estudio preliminar de Sandoval y Bizcar ${ }^{16}$, quienes reportaron una invasión de 1,7 mm.

Con respecto a la presencia de terceros molares, este estudio encontró $88 \%$ de los casos y en los controles $90,4 \%$, lo que se diferencia a lo encontrado por Cassetta et al. ${ }^{14}$, quienes observaron $89,9 \%$ en el grupo control y $100 \%$ en los casos, Sonis y Ackerman ${ }^{15}$ encontraron una frecuencia del $66.5 \%$ en los casos. Estos resultados hacen pensar que la discrepancia posterior por la presencia de tercer molar no tendría ninguna relación con la inclinación mesial del segundo molar.

En relación a la distancia entre el primer molar y el borde anterior de la rama encontramos que fue significativamente diferente entre casos y controles, lo que concuerda con lo encontrado por Shapira et al. ${ }^{2}$, Cassetta et al. ${ }^{14}$.

Se encontró que el ángulo entre el segundo molar y el plano oclusal fue significativamente diferente entre casos y controles coincidiendo con lo reportado por Shapira et al. ${ }^{2}$.

La invasión del espacio distal del primer molar por la corona inclinada del segundo molar resultó ser estadísticamente diferente entre los casos y los controles, similar a lo encontrado por Sandoval y Bizcar $^{16}$.

Shapira et al. $^{2}$ en su estudio encontró una diferencia estadísticamente significativa en el ángulo formado entre el eje axial de segundo molar y la línea del plano oclusal lo que coincide con lo observado en este estudio. Cabe señalar que Alligri et al. ${ }^{7}$ midieron la inclinación mesial del segundo molar en el tiempo mientras continuaba su formación radicular y observaron que los superiores presentaron algunas variaciones, no así los inferiores, los cuales mantuvieron una inclinación muy parecida durante su crecimiento; pudiendo pensar entonces que esta variable al presentar pocas variaciones sería factible de medir tempranamente.

La posibilidad de predecir en etapas tempranas el resultado a largo plazo de la erupción del segundo molar tiene un rol importante en el pronóstico del tratamiento temprano en ortodoncia. La metodología estadística aplicada para el análisis discriminante, resultó ser eficiente para identificar las variables predictivas. Sin embargo otros factores pueden estar influyendo en el resultado de una erupción exitosa, como puede ser tamaño dentario, tamaño de la base apical, características del tejido blando u otros que no son factibles de medir en una radiografía panorámica y que sin duda pueden ayudar a mejorar el modelo.

El modelo predictivo desarrollado en este estudio puede identificar molares con o sin probabilidad de erupción natural. Esta observación no necesariamente quiere decir que todos los pacientes sin probabilidad deban requerir una cirugía de reposicionamiento automáticamente, ya que se han desarrollado alternativas ortodóncicas que cada profesional debe valorar en su mérito de acuerdo a las condiciones de cada paciente.

Es necesario aplicar el modelo en una cohorte prospectiva para determinar la validez del mismo y hacer los ajustes necesarios, toda vez que para concebirla se utilizó una muestra de corte transversal.

Finalmente podemos concluir que existe diferencia estadísticamente significativa del ángulo del $2 \mathrm{MM}$ entre casos y controles. La distancia desde distal del $1 \mathrm{MM}$ al borde anterior de la rama mandibular fue estadísticamente significativa entre casos y controles, al igual que la inclinación mesial de $2 \mathrm{MM}$ con respecto al PO y al plano mandibular. La invasión distal del $1 \mathrm{MM}$ por el $2 \mathrm{MM}$ fue también estadísticamente significativa entre casos y controles. Por lo cual es necesario contar con nuevos estudios que nos permitan establecer claramente que variables deben ser medidas y cuáles serían los valores de normalidad para poder diagnosticar en forma precoz esta condición.

\section{CONFLICTO DE INTERESES}

Los autores declaran no tener ningún conflicto de intereses. 


\section{Bibliografía}

1. Evans R. Incidence of lower second permanent molar impaction. $\mathrm{Br} \mathrm{J}$ Orthod. 1988; 15:199-203

2. Shapira Y, Finkelstein T, Shpack N, Lai YH, Kuftinec MM, Vardimon A. Mandibular second molar impaction. Part I: Genetic traits and characteristics. Am J Orthod Dentofacial Orthop. 2011; 140:32-7.

3. Magnusson C, Kjellberg $\mathrm{H}$. Impaction and retention of second molars: diagnosis, treatment and outcome. A retrospective follow-up study. Angle Orthod. 2009 79:422-7.

4. Cho SY, Ki Y, Chu V, Chan J. Impaction of permanent mandibular second molars in ethnic Chinese schoolchildren. J Can Dent Assoc. 2008; 74:521.

5. Bondemark L, Tsiopa J. Prevalence of ectopic eruption, impaction, retention and agenesis of the permanent second molar. Angle Orthod. 2007; 77:773-8.

6. Fu PS, Wang JC, Wu YM, Huang TK, Chen WC, Tseng YC, et al. Impacted mandibular second molars. A retrospective study of prevalence and treatment outcome. Angle Orthod. 2012; 82:670-5.

7. Alligri A, Putrino A, Cassetta M, Silvestri A, Barbato E, Galluccio G. The mandibular permanent second molars and their risk of impaction: a retrospective study. Eur J Paediatr Dent. 2015; 16:246-50.

8. Lau C, Whang C, Bister D. Orthodontic uprighting of severely impacted mandibular second molars. Am J Orthod Dentofacial Orthop. 2013; 143:116-24

9. Sawicka M, Racka-Pilszak B, Rosnowska-Mazurkiewicz A. Uprighting partially impacted permanent second molars. Angle Orthod. 2007; 77:148-54.
10. Shpack N, Finkelstein T, Lai Y, Kuftinec M, Vardimon A, Shapira Y. Mandibular Permanent Second Molar Impaction Treatment options and outcome. Open Journal of Dentistry and Oral Medicine. 2013;1: 9-14.

11. Manterola C, Otzen T. Observational Studies. The Most Commonly Used Designs in Clinical Research. Int. J. Morphol. 2014; 32:634-45.

12. Rubin RL, Baccetti T, McNamara JA Jr. Mandibular second molar eruption difficulties related to the maintenance of arch perimeter in the mixed dentition. Am J Orthod Dentofacial Orthop. 2012; 141:146-52.

13. Fleiss $\mathrm{JL}$, Cohen J. The equivalence of weighted kappa and the intraclass correlation coefficient as measures of reliability. Educ Psychol Meas. 1973; 33: 613-19.

14. Cassetta M, Altieri F, Di Mambro A, Galluccio G, Barbato E. Impaction of permanent mandibular second molar: a retrospective study. Med Oral Patol Oral Cir Bucal. 2013; 18:e564-8.

15. Sonis A, Ackerman M. E-space preservation. Angle Orthod. 2011; 81:1045-9. 16. Sandoval P, Bizcar B. Probabilidad de retención de segundos molares permanentes mandibulares (Estudio piloto). Odontoestomatología. 2014; 16:39-44

17. Baccetti T, Franchi L, McNamara JA. Cephalometric variables predicting the long-term success or failure of combined rapid maxillary expansion and facial mask therapy. Am J Orthod Dentofacial Orthop. 2004.126:16-22.

18. Battagel JM. Discriminant Analysis: a model for the prediction of relapse in Class III children treated orthodontically by a non-extraction technique. Eur J Orthod. 1993; 15:199-209. 